\title{
Multi-Layer Decision Methodology For Ranking Z-Numbers
}

\author{
Ahmad Syafadhli Abu Bakar \\ School of Computing, University of Portsmouth, Buckingham Building, \\ Portsmouth, PO1 3HE, United Kingdom \\ Alexander Gegov \\ School of Computing, University of Portsmouth, Buckingham Building, \\ Portsmouth, PO1 3HE, United Kingdom \\ E-mail: alexander.gegov@port.ac.uk \\ www.port.ac.uk
}

Received 15 January 2014

Accepted 10 November 2014

\begin{abstract}
The new concept of a $Z$ - number has been recently introduced in decision making analysis. This concept is capable of effectively dealing with uncertainty in information about a decision. As this concept is relatively new in fuzzy sets, its underlying theoretical aspects have not been established yet. In this paper, a multi-layer methodology for ranking $\mathrm{Z}$ - numbers is proposed for the first time. This methodology consists of two layers: $\mathrm{Z}$ - number conversion as the first layer and fuzzy number ranking as the second layer. In this study, the conversion methodology of $Z$ - numbers into fuzzy numbers is extended to conversion into standardised generalised fuzzy number so that the methodology is applicable to both positive and negative data values. The methodology is validated by means of thorough comparison with some established ranking methods for consistency purposes. This methodology is considered as a generic decision making procedure, especially when $Z$ - numbers are applied to real decision making problems.
\end{abstract}

Keywords: $\boldsymbol{Z}$ - numbers; ranking $Z$ - numbers; consistency with human intuition; standardised generalised fuzzy numbers. 


\section{Introduction}

Fuzzy set theory serves as the basis of formal decision making analysis when uncertainty factors are involved in human decision making. This is expressed through ability of human in making logical decisions using imprecise and incomplete information which leads to uncertainty in terms of decision informativeness. Fuzzy set of numbers or fuzzy numbers are often used in various decision making situations such as risk analysis by S. Chen et al. (2012), supply chain management (D. Wu et al., 2013), fuzzy portfolio (J. Bermudez et al., 2012), selection of construction project (S. Ebrahimnejad et al., 2012) and decision making on water resources (D. Morais, A. Almeida, 2012). Main reason of fuzzy number is utilised in those decision making situations is because of its capability to appropriately deal with imprecise numerical quantities and subjective preferences of decision makers (H. Deng, 2013). It has to be noted that fuzzy numbers are represented by possibility distribution (H. Zimmerman, 2000; A. Kumar et al., 2010), thus influence practitioners to compare or rank them rather than evaluate them directly for effective decision making in specific circumstances.

Concept of ranking fuzzy number introduces by $\mathrm{R}$. Jain (1976) attracts special attention due to their wide applications in theory of fuzzy decision making and fuzzy data analysis. In order to achieve greater efficiencies and accuracies in ranking results, several ranking methods are recently suggested in literature. Among them are S. Chen, J. Chen (2009), A. Bakar et al. (2010), S. Chen, K. Sanguatsan (2011), A. Bakar et al. (2012), L. Dat et al. (2012) and V. Yu et al. (2013). It is worth mentioning here that main processes involve in ranking fuzzy numbers are evaluation of a fuzzy number and comparison with other fuzzy numbers under consideration. Nonetheless, these are not easy processes as fuzzy numbers are represented by possibility distributions, imply that they are overlapped with each other. Therefore, determining clearly a fuzzy number is larger or smaller than another is a difficult task.

It is worth expressing here that a new ranking fuzzy numbers method is proposed by authors in A. Bakar, A. Gegov (2014) whereby this method uses centroid point and spread (CPS) in ranking fuzzy numbers. Capabilities of this method in ranking fuzzy numbers are shown when the method solves problems faced by recently established ranking methods of S. Chen, J. Chen (2009), A. Kumar et al. (2010), S. Chen, K. Sanguatsan (2011) and L. Dat et al. (2012) in ranking embedded fuzzy numbers of different shapes but having same centroid, embedded fuzzy numbers of different spread and embedded fuzzy numbers of different normality respectively. Even though, CPS ranking method capable to solve shortcomings of existing established ranking methods, limitation of fuzzy numbers to appropriately representing reliability of information in many practical situations (B. Kang et al., 2012a), affect indirectly the usefulness of utilising ranking fuzzy numbers method in decision making.

Issue with regards to reliability of information is important in decision making environment (B. Kang et al., 2012a, b) as this is extensively discussed in L. Zadeh (2011). In the discussion, a new concept, $Z$ numbers is introduced to cater the limitation of fuzzy numbers. According to L. Zadeh (2011), Z - number is considered as generalisation of numbers, intervals, fuzzy numbers and random numbers because it is ranked with the highest level of generality, level 3 compared to numbers (ground level 1), intervals (level 1), fuzzy numbers (level 2) and random numbers (level 2). It is worth mentioning here that if level of generality of a number is higher than others, then capability of a number to construct realistic model of real world system is greater than others (L. Zadeh, 2011). In this case, Znumbers are having better representation than fuzzy numbers.

Although, the aforementioned evidences show that $Z$ - number is more applicable than fuzzy numbers, discussion in terms of $Z$ - number is few and inadequate in the literature. This is because, $Z-$ number is relatively a new concept introduced in literature thus theoretical view on this concept is not fully established. Some theoretical frameworks on $\mathrm{Z}$ - numbers are only done by R. Yager (2012a,b), B. Kang et al. (2012b) and L. Gardashova (2014) on relationship between Z numbers and linguistic knowledge, involvement $\mathrm{Z}$ numbers in uncertain decision making environment and utilisation of $\mathrm{Z}$ - numbers on multi - criteria decision making respectively. According to L. Zadeh (2011), since nature of $Z$ - numbers is more complex than fuzzy numbers, hence one way to effectively deal with $Z-$ numbers is by reducing a $Z$ - number to a certain level of number generality without losing informativeness about the computational results. In this respect, B. Kang et al. (2012) come out with a method that converts $Z$ numbers into fuzzy number which is based on Fuzzy Expectation of a fuzzy set.

Since, $\mathrm{Z}$ - numbers are more applicable than fuzzy numbers and recent work by B. Kang et al. (2012a) proposes a method for decision making purposes, hence this paper suggests a multi - layer methodology for ranking $\mathrm{Z}$ - numbers as a decision making methodology when $Z$ - number is used. It has to be noted here that this methodology consists of two layers namely $Z$ numbers conversion method by B. Kang et al. (2012a) as layer one and CPS ranking method (A. Bakar, A. Gegov, 2014) as layer two. In this methodology, B. Kang et al. (2012a) work on converting $\mathrm{Z}$ - numbers 
into fuzzy numbers is extended to conversion of $\mathrm{Z}-$ numbers into standardized generalised fuzzy numbers due to the existence of both positive and negative data values in real decision making problems. Overall, this methodology is considered as a complete, generic methodology when $Z$ - numbers are used in decision making applications.

The remainder of the paper is organised as follows: Section 2 discusses theoretical preliminaries of this study, Section 3 covers on methodology of previous established works, Section 4 provides details on development of the proposed work, Section 5 illustrates validation of the proposed work and conclusion is given in Section 6.

\section{Theoretical Preliminaries}

Based on S. Chen, J. Chen (2009), some basic concepts used in this paper are illustrated as follows.

\subsection{Trapezoidal Fuzzy Numbers}

A trapezoidal fuzzy numbers is represented by the following membership function given by

$$
\mu_{A}(x)=\left(a_{1}, a_{2}, a_{3}, a_{4}\right)=\left\{\begin{array}{ccc}
\frac{x-a_{1}}{a_{2}-a_{1}} & \text { if } & a_{1} \leq x \leq a_{2} \\
1 & \text { if } & a_{2} \leq x \leq a_{3} \\
\frac{a_{4}-x}{a_{4}-a_{3}} & \text { if } & a_{3} \leq x \leq a_{4} \\
0 & \text { otherwise }
\end{array}\right.
$$

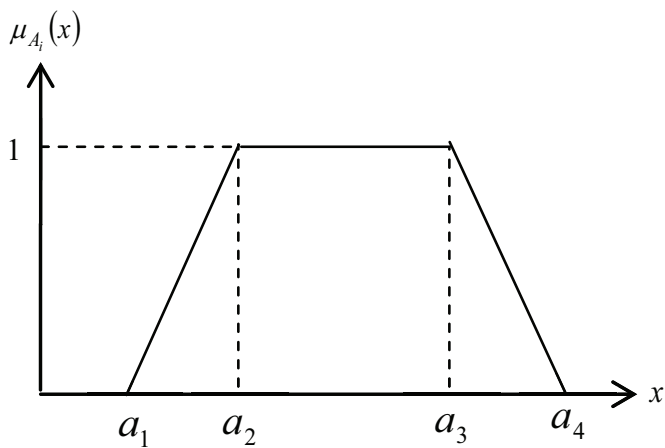

Fig 1: Trapezoidal Fuzzy Number

For a trapezoidal fuzzy number, if $a_{2}=a_{3}$, then the fuzzy number is in the form of triangular fuzzy number. Using this condition, if $a_{1}=a_{2}=a_{3}=a_{4}$ for both triangular and trapezoidal fuzzy numbers, then both fuzzy numbers are in the form of singleton fuzzy number (crisp value). It has to be noted here that length between $a_{1}$ and $a_{4}$ is core of the fuzzy number.

\subsection{Generalised Trapezoidal Fuzzy Numbers}

A fuzzy number, $A=\left(a_{1}, a_{2}, a_{3}, a_{4} ; w_{A}\right)$ is a generalised trapezoidal fuzzy number when $a_{1}, a_{2}, a_{3}$, $a_{4}$ are real numbers and $w_{A}$ which represents the height of fuzzy number $A$ is in interval of $[0,1], w_{A} \in[0,1]$. Similarly as in $2(A)$, whenever $a_{2}=a_{3}, A$ is known as a generalised triangular fuzzy numbers (S. Chen, J. Chen, 2009).

A generalised trapezoidal fuzzy numbers is represented by the following membership function given by

$$
\mu_{A}(x)=\left(a_{1}, a_{2}, a_{3}, a_{4}\right)=\left\{\begin{array}{ccc}
\frac{x-a_{1}}{a_{2}-a_{1}} & \text { if } & a_{1} \leq x \leq a_{2} \\
w_{A} & \text { if } & a_{2} \leq x \leq a_{3} \\
\frac{a_{4}-x}{a_{4}-a_{3}} & \text { if } & a_{3} \leq x \leq a_{4} \\
0 & \text { otherwise }
\end{array}\right.
$$

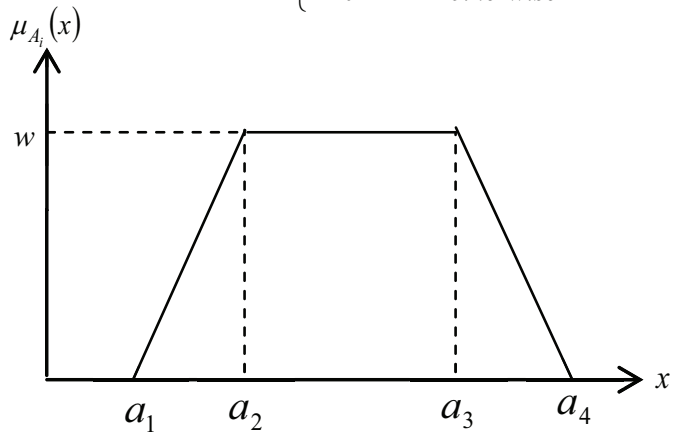

Fig 2: Generalised Trapezoidal Fuzzy Number

\subsection{Standardised Generalised Trapezoidal Fuzzy Numbers}

If fuzzy number $A$ has the property such that -1 $<a_{1}<a_{2}<a_{3}<a_{4}<1$ then $A$ is called as a standardized generalised trapezoidal fuzzy number (S. Chen, J. Chen, 2009) and is denoted as

$$
\widetilde{A}=\left(\widetilde{a}_{1}, \widetilde{a}_{2}, \widetilde{a}_{3}, \widetilde{a}_{4} ; w_{\widetilde{A}}\right)
$$

Furthermore if $\widetilde{a}_{2}=\widetilde{a}_{3}$, then $\widetilde{A}$ is a standardized generalised triangular fuzzy number. Any generalised fuzzy number is transformed into standardized 
generalised fuzzy numbers using normalisation process described as

$$
\begin{aligned}
\widetilde{A} & =\left(\frac{a_{1}}{k}, \frac{a_{2}}{k}, \frac{a_{3}}{k}, \frac{a_{4}}{k} ; w_{A}\right) \\
& =\left(\widetilde{a}_{1}, \widetilde{a}_{2}, \widetilde{a}_{3}, \widetilde{a}_{4} ; w_{\widetilde{A}}\right)
\end{aligned}
$$

where $k=\max \left(a_{1}, a_{2}, a_{3}, a_{4}\right)$.

It has be noted that normalisation process in (2) affects only on components of fuzzy numbers such that $a_{1}, a_{2}, a_{3}, a_{4}$ are changed into $\widetilde{a}_{1}, \widetilde{a}_{2}, \widetilde{a}_{3}, \widetilde{a}_{4}$ while height of the fuzzy number, $w_{A}$ remains the same (S. Chen, J. Chen, 2009).

\section{4. $Z$ - numbers}

According to L. Zadeh (2011), a $Z$ - number is an ordered pair of fuzzy number denoted as $Z=(\bar{A}, \bar{B})$. First component, $\bar{A}$ is known as restriction component whereby it is a real - valued uncertain on $X$ while second component, $\bar{B}$ is a measure of reliability for $\bar{A}$. Illustration for $Z$ - number is depicted in Fig 2 (Kang et al.) shows as follows.

A $\mathrm{Z}$ - number is represented by the following membership functions given by

$$
\begin{gathered}
\mu_{\bar{A}}(x)=\left(a_{1}, a_{2}, a_{3}, a_{4}\right)=\left\{\begin{array}{ccc}
\frac{x-a_{1}}{a_{2}-a_{1}} & \text { if } & a_{1} \leq x \leq a_{2} \\
1 & \text { if } & a_{2} \leq x \leq a_{3} \\
\frac{a_{4}-x}{a_{4}-a_{3}} & \text { if } & a_{3} \leq x \leq a_{4} \\
0 & \text { otherwise }
\end{array}\right. \\
\mu_{\bar{B}}(x)=\left(b_{1}, b_{2}, b_{3}, b_{4}\right)=\left\{\begin{array}{ccc}
\frac{x-b_{1}}{b_{2}-b_{1}} & \text { if } & b_{1} \leq x \leq b_{2} \\
1 & \text { if } & b_{2} \leq x \leq b_{3} \\
\frac{b_{4}-x}{b_{4}-b_{3}} & \text { if } & b_{3} \leq x \leq b_{4} \\
0 & \text { otherwise }
\end{array}\right.
\end{gathered}
$$
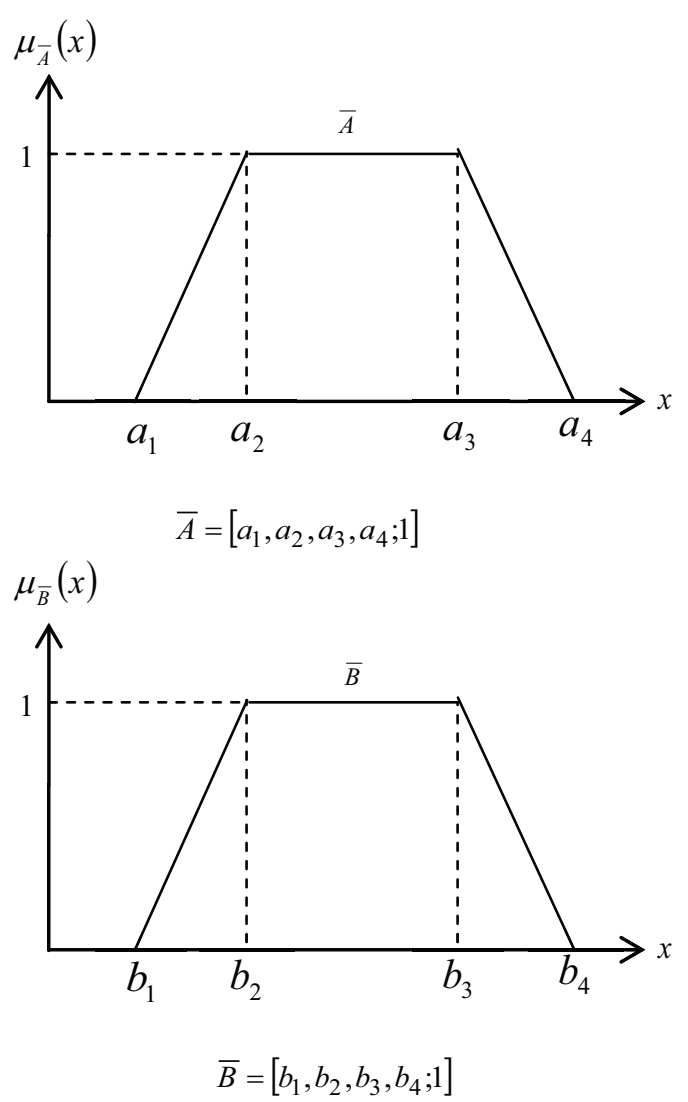

Fig 3.A $Z$ - number, $Z=(\bar{A}, \bar{B})$

\section{Previous Works}

In this section, details with regards to methods used in developing the proposed multi - layer methodology are shown. Full descriptions on the methods are as follows:

\subsection{Z-Numbers Conversion Method}

Suggestion by L. Zadeh (2011) on reducing $Z$ number to another representation without losing its computational informativeness, leads B. Kang et al. (2012a) to presenting a method of converting $\mathrm{Z}$ numbers into fuzzy numbers. Based on B. Kang et al. (2012a), complete steps of the method are as follows.

Step 1: Convert reliability component, $\bar{B}$ into a crisp number, $\alpha$ using the following equation 


$$
\alpha=\frac{\int_{-\infty}^{\infty} x \mu_{B}^{-}(x) d x}{\int_{-\infty}^{\infty} \mu_{B}(x) d x}
$$

Note that, $\alpha$ represents the weight of the reliability component of $\mathrm{Z}$ - number.

Step 2: Add $\alpha$ to restriction component, $\widetilde{A}$ to form a weighted restriction of $\mathrm{Z}$ - number which is denoted as

$$
\widetilde{Z}^{\alpha}=\left\{\left\langle x, \mu \widetilde{A}^{\alpha}(x)\right\rangle \mid \mu \widetilde{A}^{\alpha}(x)=\alpha \mu_{\widetilde{A}}(x), x \in[0,1]\right\}
$$

Step 3: Convert $\widetilde{Z}^{\alpha}$ into fuzzy numbers which is represented as

$$
\widetilde{Z}^{\prime}=\left\{\left\langle x, \mu_{\widetilde{Z}^{\prime}}(x)\right\rangle \mid \mu_{\widetilde{Z}^{\prime}}(x)=\mu \widetilde{A}\left(\frac{x}{\sqrt{\alpha}}\right), x \in[0,1]\right\}
$$

Based on B. Kang et al. (2012a) work, it is shown that process of converting $Z$ - numbers into fuzzy numbers is sensible and logical because result obtained by the study shows $Z$ - number is reduced to a lower level of generality, $Z$ - number into fuzzy number and computational informativeness remains unchanged. Moreover, the conversion of $\mathrm{Z}$ - number into fuzzy number is reasonable due to the fact that both $\widetilde{Z}^{\alpha}$ and $\tilde{Z}^{\prime}$ are same when Fuzzy Expectation Theorem is applied.

\subsection{Ranking of Fuzzy Numbers Based on Centroid Point and Spread}

As mentioned in the introduction section, CPS ranking method is introduced in A. Bakar \& A. Gegov (2014) to cater limitations faced by existing established methods in ranking fuzzy numbers. Effectiveness of this method is proved when this method capable to rank various fuzzy numbers cases in practical use. Illustration with regards to full methodology of CPS ranking method is as follows:

Assume that a fuzzy number $A$ is generally described as $A=\left(a_{1}, a_{2}, a_{3}, a_{4} ; w_{A}\right)$,

Step 1: Compute centroid point value for fuzzy number $A$ using B. Shieh (2007) formula such that horizontal $-x$ centroid of $A, x_{A}$ is calculated as

$$
x_{A}=\frac{\int_{-\infty}^{\infty} x f(x) d x}{\int_{-\infty}^{\infty} f(x) d x}
$$

and vertical $-y$ cenrtroidof $A, y_{A}$ is

$$
y_{A}=\frac{\int_{0}^{w_{A_{i}}} \alpha\left|A_{i}^{\alpha}\right| d \alpha}{\int_{0}^{w_{A_{i}}}\left|A_{i}^{\alpha}\right| d \alpha}
$$

where

$\left|A_{i}^{\alpha}\right|$ is length of $\alpha$-cuts of fuzzy number $A, x_{A} \in[-1$, 1] and $y_{A} \in\left[0, w_{A}\right]$.

Step 2: Calculate spread value for fuzzy number $A$ by considering

distance along $x-$ axis from $x_{A}$ defines as

$$
\begin{aligned}
i_{A} & =\operatorname{dist}\left(a_{4}-a_{1}\right)=\left|a_{4}-x_{A}\right|+\left|x_{A}-a_{1}\right| \\
& =\left|a_{4}-a_{1}\right|
\end{aligned}
$$

distance along vertical $y$ - axis $y_{A}$ defines

$$
i i_{A}=y_{A}
$$

Therefore, spread of $A, s_{A}$ is defined as

$$
s_{A}=i_{A} \times i i_{A}
$$

where $i$ and $i i$ are $\operatorname{dist}\left(a_{4}-a_{1}\right)$ and $y_{A}$ respectively.

$s_{A}, i_{A}, i i_{A}, \operatorname{dist}\left(a_{4}-a_{1}\right) \in[0,1]$.

Step 3: Determine the ranking value for each fuzzy number under consideration using the following equation as

$$
\operatorname{CPS}(A)=x_{A}^{*} \times y_{A}^{*} \times\left(1-s_{A}\right)
$$

where

$x_{A}^{*}$ is the horizontal $-x$ centroid for fuzzy number $A$

$y_{A}^{*}$ is the vertical $-y$ centroid for fuzzy number $A$

$s_{A}$ is the spread for fuzzy number $A$

$\operatorname{CPS}(A) \in[-1,1]$ 
If $C P S(A)>C P S(B)$, then $A \succ B$ (i.e. $A$ is greater than $B$ ).

If $C P S(A)<C P S(B)$, then $A \prec B$ (i.e. $A$ is lesser than $B$ ).

If $C P S(A)=C P S(B)$, then $A \approx B$ (i.e. $A$ and $B$ are equal).

It is understandable that every presented method of ranking fuzzy numbers has their own advantages and disadvantages. Nonetheless, CPS ranking method proves its capability by ranking various fuzzy numbers cases with correct ranking order such that the ranking results are consistent with human intuition. Therefore, using CPS ranking method as provides great assistance towards decision makers in solving various decision making problems.

\section{Proposed Methodology}

As discussed in introduction section, literature on $\mathrm{Z}$ - numbers is inadequate which indicates that dealing $Z$ - numbers using current knowledge is ineffective. In this section, a multi - layer decision making methodology for ranking $Z$ - numbers is illustrated. It has to be noted here that the methodology consists of two layers namely

- Layer One: $\mathrm{Z}$ - numbers conversion method (B. Kang et al., 2012a).

- Layer Two: CPS ranking method (A. Bakar \& A. Gegov, 2015).

Full description for both layers is as follows:

\subsection{Layer One}

Step A1: Convert reliability component, $\bar{B}$ into a crisp number, $\alpha$ (weight of the reliability component) using (3)

Step A2: Add $\alpha$ to restriction component, $\bar{A}$ to form weighted restriction of $Z$ - number as in (4).

Step A3: Convert the weighted restriction of $\mathrm{Z}-$ number into standardised generalised fuzzy numbers as in (5).
It has to be noted that Step 1 till Step 3 of Layer One are the same as in Section $3(A)$. However, it is worth mentioning here that in Step 3 of Layer One, fuzzy number used in Section $3(A)$ is extended to standardised generalised fuzzy numbers as defines in Section $2(C)$.

\subsection{Layer Two}

Let $Z_{A}=\left[z_{a 1}, z_{a 2}, z_{a 3}, z_{a 4}\right]$ be $\quad$ standardised generalised fuzzy number obtained from Layer One known as $Z$ - fuzzy number $A$ and description for Layer Two is as follows:

It has to be noted that, a $Z$ - fuzzy number, $Z_{A}$ refers to standardised generalised fuzzy number after conversion from Z - number in Layer One.

Step B1: Compute centroid point for $Z_{A}$ by finding horizontal $-x$ centroid as

$$
x_{z_{A}}^{*}=\frac{\int_{-\infty}^{\infty} x f(x) d x}{\int_{-\infty}^{\infty} f(x) d x}
$$

and vertical $-y$ centroid as

$$
y_{Z_{A}}^{*}=\frac{\int_{0}^{w_{Z} A_{i}} \alpha\left|Z_{A_{i}^{\alpha}}\right| d \alpha}{\int_{0}^{w_{Z}}{ }_{A_{i}}\left|Z_{A_{i}^{\alpha}}\right| d \alpha}
$$

where

$$
\begin{aligned}
& \left|Z_{A_{i}^{\alpha}}\right| \text { is length of } \alpha \text {-cuts of } Z_{A} \\
& x_{Z_{A}}^{*} \in[-1,1] \text { and } y_{Z_{A}}^{*} \in\left[0, w_{Z_{A_{i}}}\right] .
\end{aligned}
$$

Step B2: Calculate spread value for $Z_{A}$ by considering distance along $x$-axis from $x_{z_{A}}^{*}$ defines as

$$
\begin{aligned}
i_{z_{A}} & =\operatorname{dist}\left(z_{a 4}-z_{a 1}\right) \\
& =\left|z_{a 4}-x_{z_{A}}^{*}\right|+\left|x_{z_{A}}^{*}-z_{a 1}\right| \\
& =\left|z_{a 4}-z_{a 1}\right|
\end{aligned}
$$

distance along the vertical $y$ - axis from $y_{z_{A}}^{*}$ defines as

$$
i i_{z_{A}}=y_{z_{A}}^{*}
$$

Therefore, spread of $Z_{A}, s_{Z_{A}}$ is defined as 


$$
s_{Z_{A}}=i_{Z_{A}} \times i i_{Z_{A}}
$$

where $i_{z_{A}}$ and $i i_{z_{A}}$ are $\operatorname{dist}\left(z_{a 4}-z_{a 1}\right)$ and $y_{z_{A}}^{*}$ respectively.

$s_{Z_{A}}, i_{Z_{A}}, i i_{Z_{A}}, \operatorname{dist}\left(z_{a 4}-z_{a 1}\right) \in[0,1]$.

Step B3: Determine ranking value for each Z - fuzzy numbers under consideration using the following equation as

$$
\operatorname{CPS}_{Z}\left(Z_{A}\right)=x_{z_{A}}^{*} \times y_{z_{A}}^{*} \times\left(1-s_{z_{A}}\right)
$$

where

$x_{Z_{A}}^{*}$ is the horizontal $-x$ centroid for $Z_{A}$

$y_{z_{A}}^{*}$ is the vertical $-y$ centroid for $Z_{A}$

$S_{Z_{A}}$ is the spread for $Z_{A}$

$\operatorname{CPS}_{Z}\left(Z_{A}\right) \in[-1,1]$

If $C P S_{Z}\left(Z_{A}\right)>C P S_{Z}\left(Z_{B}\right)$, then $Z_{A} \succ Z_{B}$. (i.e. $Z_{A}$ is greater than $\left.Z_{B}\right)$.

If $C P S_{Z}\left(Z_{A}\right)<C P S_{Z}\left(Z_{B}\right)$, then $Z_{A} \prec Z_{B}$. (i.e. $Z_{A}$ is lesser than $\left.Z_{B}\right)$.

If $C P S_{Z}\left(Z_{A}\right)=C P S_{Z}\left(Z_{B}\right)$, then $Z_{A} \approx Z_{B}$. (i.e. $Z_{A}$ and $Z_{B}$ are equal).

It has to be noted here that all steps in Layer Two are similar as in Section $3(B)$ but the only distinction between Section $3(B)$ and Layer Two is utilisation of $Z$ - fuzzy numbers and fuzzy numbers in the formulations respectively.

\section{Validation of Results}

In this section, validation for the proposed multi layer methodology for ranking $\mathrm{Z}$ - numbers is provided. Since, the proposed methodology consists of two layers, hence validations of the proposed work are as well in two parts. Complete validations processes of the proposed methodology are as follow:

\subsection{Layer One}

Let $A=\left(a_{1}, a_{2}, a_{3}, a_{4}, 1\right)$ and $R=\left(r_{1}, r_{2}, r_{3}, 1\right)$ be two standardized generalised fuzzy numbers representing expert's opinion and its reliability respectively

where $\mathrm{Z}$ - number, $Z=(A, R)$ such that $A, R \in[-1,1]$.

Using Definition and Section 2(D), expert's opinion and its reliability are expressed using $\mathrm{Z}$ - numbers depicted as

$$
Z=\left[\left(a_{1}, a_{2}, a_{3}, a_{4} ; 1\right),\left(r_{1}, r_{2}, r_{3} ; 1\right)\right]
$$

Based on equation (1), expert's reliability component, $R=\left(r_{1}, r_{2}, r_{3} ; 1\right)$, is converted into crisp value, $\alpha_{R}$, where $\alpha_{R}$ is then added to constraint component, $A$ to form weighted $\mathrm{Z}$ - number, $Z_{\alpha}$ signifies as follows:

$$
Z_{\alpha}=\left(a_{1}, a_{2}, a_{3}, a_{4} ; \alpha_{R}\right)
$$

\section{Proposition 1}

According to B. Kang et al. (2012a), the following are obtained when Fuzzy Expectation theorem is used

$$
\begin{array}{ll} 
& E_{A^{\alpha}}(x)=\alpha E_{A}(x), x \in X \\
\text { subject to } \quad & \mu_{A^{\alpha}}(x)=\alpha \mu_{A}(x), x \in X
\end{array}
$$

Proof

$$
E_{\tilde{A}^{\alpha}}(x)=\int_{X} x \mu_{\tilde{A}^{\alpha}}(x) d x=\int_{X} \alpha x \mu \mu_{\tilde{A}}(x) d x=\alpha \int_{X} x \mu \tilde{\tilde{A}}(x) d x=\alpha E_{\tilde{A}}(x)
$$

Using the same theorem, weighted $Z$ - number, $Z_{\alpha}$ is converted into standardized generalised fuzzy number which is shown as follows

$$
Z^{*}=\left(\sqrt{\alpha_{R}} \times a_{1}, \sqrt{\alpha_{R}} \times a_{2}, \sqrt{\alpha_{R}} \times a_{3}, \sqrt{\alpha_{R}} \times a_{4} ; 1\right)
$$

where $\quad Z^{*} \in[-1,1]$ such that $a_{1}, a_{2}, a_{3}, a_{4} \in[-1,1]$, $\alpha_{R} \in[0,1]$.

Proposition 2

$\begin{array}{ll} & E_{Z^{*^{\prime}}}(x)=\alpha E_{\widetilde{A}}(x), x \in \sqrt{\alpha} X \\ \text { subject to } & \mu_{Z^{*}}(x)=\mu_{\widetilde{A}}\left(\frac{x}{\sqrt{\alpha}}\right), x \in \sqrt{\alpha} X\end{array}$ 
Proof

$$
\begin{aligned}
E_{Z^{*}}(x) & =\int_{\sqrt{\alpha} X} x \mu_{Z^{*}}(x) d x=\int_{\sqrt{\alpha} X} x \mu_{A}\left(\frac{x}{\sqrt{\alpha}}\right) d x x \\
& =\sqrt{\alpha} t \int_{X}(\sqrt{\alpha} t) \mu_{A}(t) d(\sqrt{\alpha} t)=\alpha \int_{X} t \mu_{A}(t) d t=\alpha E_{A}(x)
\end{aligned}
$$

Theoretical proof completion in terms utilizing Fuzzy Expectation Theorem on conversion of $Z$ - numbers into standardized generalised fuzzy numbers is shown by the following proposition.

\section{Proposition 3}

$$
E_{Z^{z^{*}}}(x)=E_{A^{\alpha}}(x)
$$

\section{Proof}

It has to be noted that from equation (18) and equation (20), the following are obtained

$$
\begin{gathered}
E_{A^{\alpha}}(x)=\alpha E_{A}(x) \\
E_{Z^{*}}(x)=\alpha E_{A^{\alpha}}(x) \\
E_{Z^{*}}(x)=E_{A^{\alpha}}(x)
\end{gathered}
$$

\section{A. Layer Two}

Since, $\mathrm{Z}$ - fuzzy numbers are fuzzy numbers, hence validation for Layer Two on $\mathrm{Z}$ - fuzzy numbers is similar as validation for fuzzy numbers. It has to be noted here that validation in this section is a comparative - based analysis which compares CPS ranking method with some established ranking methods ranking $Z$ - fuzzy numbers to ranking $Z$ - fuzzy number. It is worth emphasising here that all established ranking methods used in this section are added ' $Z$ ' (e.g. $Z$ - C. Cheng (1998)) to signify that the method is applied to ranking $Z$ - fuzzy number. It is also worth mentioning here that all ranking methods used in this validation are applied to ranking $Z$ - fuzzy numbers for the first time. The following are cases of $Z$ - fuzzy numbers which are introduced for the first time in this study that possibly represent real decision making situations.

Case 1: Embedded Z - fuzzy numbers of different shapes

Consider two $Z$ - fuzzy numbers $Z_{A}$ and $Z_{B}$ as shown in Figure 4. Correct ranking order of $Z$ - fuzzy numbers that the result is consistent with human intuition for this case is this case is $Z_{A} \succ B$ because centroid value of $Z_{A}$ is greater than $Z_{B}$. Based on Table $1, \mathrm{~S}$. Chen, J. Chen (2009) ranking method produces unreasonable ranking order such that the ranking result is inconsistent with human intuition $\left(Z_{B} \succ Z_{A}\right)$ since the method treats $Z$ fuzzy numbers with smaller centroid value as greater than the other. S. Chen, K. Sanguansat (2011) ranking method on the other hand treats both $\mathrm{Z}$ - fuzzy numbers as equal $\left(Z_{A} \approx Z_{B}\right)$, which also reflects as result that is inconsistent with human intuition. Same ranking order as S. Chen, K. Sanguansat (2011) are obtained by Cheng (1998), C. Chu, C. Tsao (2002), and A. Kumar et al. (2010). This is indicating that these methods are incapable to differentiate $\mathrm{Z}$ - fuzzy numbers appropriately. Using CPS ranking method, a correct ranking order is produced such that the result is consistent with human intuition, $Z_{A} \succ Z_{B}$. Same result is also obtained by L. Dat et al. (2012) whereby the method ranks $Z$ - fuzzy numbers with higher centroid value with higher ranking order.

Case 2: Embedded $\mathrm{Z}$ - fuzzy numbers of different spreads

Consider two $Z$ - fuzzy numbers $Z_{A}$ and $Z_{B}$ as shown in Figure 5. Correct ranking order of $Z$ - fuzzy numbers such that the result is consistent with human intuition for this case is $Z_{B} \succ Z_{A}$. This is due to the fact that ranking order for $Z$ - fuzzy number with smaller spread value is considered as greater than the other $Z$ numbers under consideration provided that all centroid values for each $\mathrm{Z}$ - fuzzy numbers under consideration are the same. Based on Table 2, C. Cheng (1998), C. Chu, C. Tsao (2002), Kumar et al. (2010), T. Allahviranloo, R. Saneifard (2012) and L. Dat et al. (2012) ranking methods are incapable to differentiate the $\mathrm{Z}$ - fuzzy numbers because they all are producing equal ranking $\left(Z_{A} \approx Z_{B}\right)$ such that the ranking results are consistent with human intuition. Ranking method by V. $\mathrm{Yu}$ et al. (2013) which utilises degree of optimisms, ranks $Z$ - fuzzy numbers according to actual decision makers' preferences. Thus, different ranking ordering is obtained by V. Yu et al. (2013) in this case. Correct ranking order such that the result is consistent with human intuition is produced by S. Chen, J. Chen (2009), S. Chen, K. Sanguansat (2011) and CPS ranking method whereby all of them rank $Z$ - fuzzy numbers by prioritise $Z$ - fuzzy numbers with lower spread as higher ranking order.

Case 3: Non - overlapping Z - fuzzy numbers of different shapes 
Consider different shapes case of two non overlapping $Z$ - fuzzy numbers $Z_{A}$ and $Z_{B}$ shown in Figure 6, the correct ranking order such that the result is consistent with human intuition is $Z_{B} \succ Z_{A}$. Apart from that, another reason of $Z_{B} \succ Z_{A}$ is because a crisp value is treated greater than any $Z$ - fuzzy numbers under consideration. Based on Table 3, only certain ranking methods are capable to ranking this $\mathrm{Z}$ - fuzzy numbers case correctly such that the result is consistent with human intuitions. Among them are S. Chen, J. Chen (2009), S. Chen, K. Sanguansat (2011), L. Dat et al. (2012) and the CPS method.

Case 4: Reflection of $Z$ - fuzzy numbers.

Consider reflection case of two non - overlapping $Z$ - fuzzy numbers $Z_{A}$ and $Z_{B}$ as shown in Figure 7 . It is obvious that $Z_{B}$ is ranked higher than $Z_{A}$ since $Z_{B}$ is situated on the farthest right compared to $Z_{A}$. Thus, correct ranking order such that the result is consistent with human intuitions is $Z_{B} \succ Z_{A}$. Based on Table 4, C. Cheng (1998) and A. Kumar et al. (2010) ranking methods are incapable to differentiate both Z - fuzzy numbers appropriately, thus producing inconsistent ranking order. Using CPS method, similar ranking ordering is obtained as C. Chu, C. Tsao (2002), S. Chen, J. Chen (2009), S. Chen, K. Sanguansat (2011), T. Allahviranloo, R. Saneifard (2012) and L. Dat et al. (2012) where all of them rank Z - fuzzy numbers of this case correctly such that the result is consistent with human intuitions, $Z_{A} \prec Z_{B}$.

It is noticeable that each ranking method considered in this study demonstrates its own strengths and weaknesses in ranking $Z$ - fuzzy numbers. Although all methods used for comparing $Z$ - fuzzy numbers in this study are actually methods for ranking fuzzy numbers, analysis in this section is provided to signify the capability of established ranking methods to not only correctly rank fuzzy numbers but also to rank $\mathrm{Z}$ - fuzzy numbers effectively.

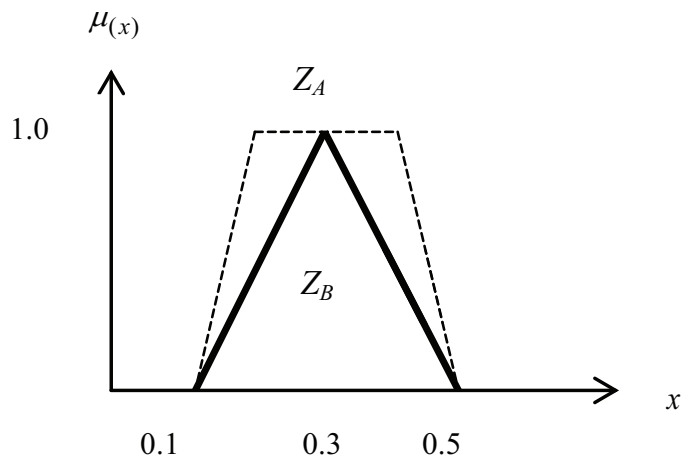

Fig $4 . Z$ - fuzzy numbers $Z_{A}$ and $Z_{B}$ of Case 1.

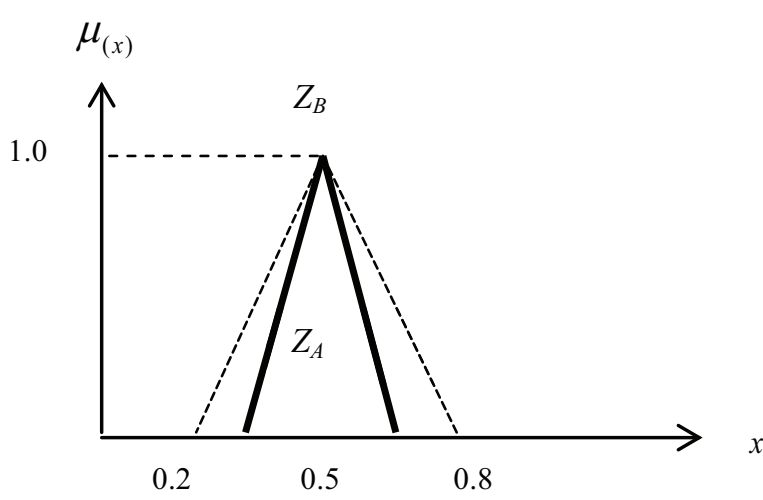

Fig 5. $Z$ - fuzzy numbers $Z_{A}$ and $Z_{B}$ of Case 2 .

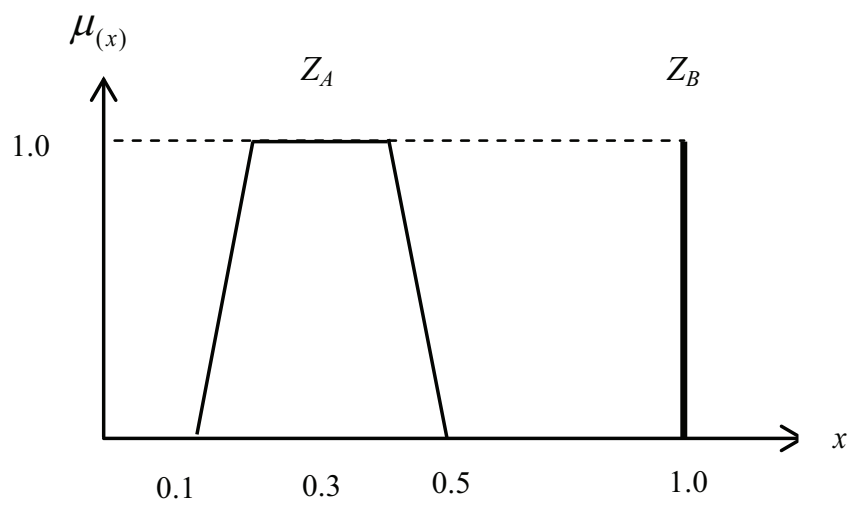

Fig $6 . Z$ - fuzzy numbers $Z_{A}$ and $Z_{B}$ of Case 3 .

Based on the analysis made above, some ranking methods dealt with cases of $Z$ - fuzzy numbers effectively while others produced irrelevant results for certain cases. Nevertheless, in each case examined above, the CPS ranking method produces effective results by giving correct ranking order for all cases of $\mathrm{Z}$ - fuzzy numbers in this study such that all results obtained are consistent with human intuition. Lists of items

\section{Conclusion}

This study proposes a novel method for ranking $\mathrm{Z}$ numbers which consists of two layers: $Z$ - numbers conversion method and CPS ranking method. The proposed multi - layer methodology is validated using both theoretical and empirical validation where results obtained from this validation are consistent with human intuition.

Furthermore, substitution of fuzzy numbers with standardised generalised fuzzy numbers in this study improves capability of $Z$ - numbers when they are applied to decision making problems. 
Although aforementioned evidence illustrates significant advantages of the proposed methodology, there are some limitations such as incapability of ranking more than two $\mathrm{Z}$ - numbers simultaneously and considering non - normal case of $Z$ - numbers (height not equal to one). This is because pairwise ranking is utilised and only normal cases of $\mathrm{Z}$ - numbers are considered in this study.
In conclusion, the proposed multi - layer decision methodology for ranking $\mathrm{Z}$ - numbers is based on intuition focused concepts for ranking $Z$ - numbers and decision making analysis. Therefore, this methodology can be further improved by other decision making studies with the purpose of making it applicable to ranking more complex cases of $Z$ - numbers.

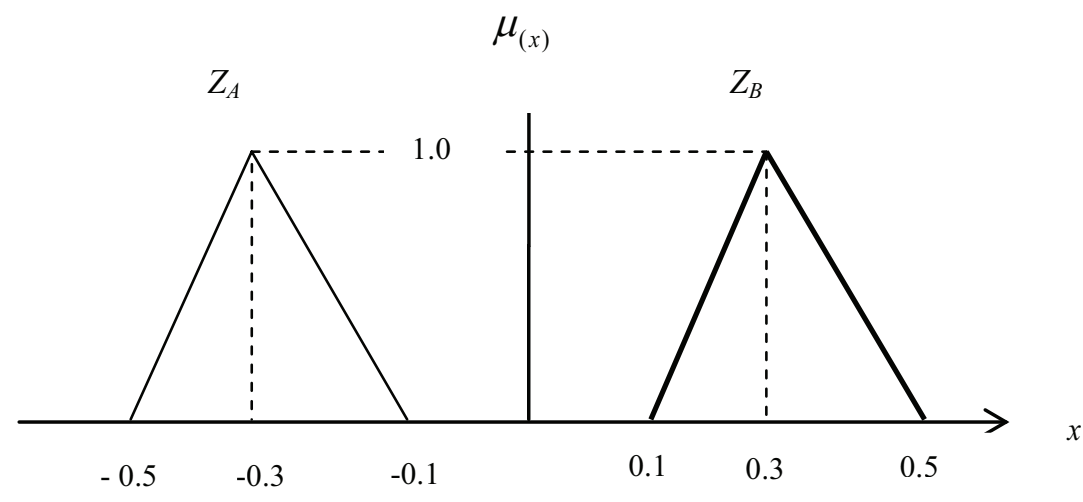

Fig $7 . Z$ - fuzzy numbers $Z_{A}$ and $Z_{B}$ of Case 4.

Table 1.Comparative results using $\mathrm{Z}$ - fuzzy numbers of Case 1 .

\begin{tabular}{|c|c|c|c|c|}
\hline \multirow[b]{2}{*}{ Methods } & \multicolumn{2}{|c|}{$\mathrm{Z}$ - fuzzy numbers } & \multirow{2}{*}{$\begin{array}{l}\text { Ranking } \\
\text { Results }\end{array}$} & \multirow[b]{2}{*}{ Evaluation } \\
\hline & $Z_{A}$ & $Z_{B}$ & & \\
\hline Z - C. Cheng (1998) & 0.583 & 0.583 & $Z_{A} \approx Z_{B}$ & Inconsistent \\
\hline Z - C. Chu, C. Tsao (2002) & 0.150 & 0.150 & $Z_{A} \approx Z_{B}$ & Inconsistent \\
\hline Z - S. Chen, J. Chen (2009) & 0.254 & 0.258 & $Z_{A} \prec Z_{B}$ & Inconsistent \\
\hline Z - A. Kumar et al. (2010) & 0.300 & 0.300 & $Z_{A} \approx Z_{B}$ & Inconsistent \\
\hline Z - S. Chen, K. Sanguansat (2011) & 0.300 & 0.300 & $Z_{A} \approx Z_{B}$ & Inconsistent \\
\hline Z - L. Dat et al. (2012) & 0.333 & 0.222 & $Z_{A} \succ Z_{B}$ & Consistent \\
\hline $\mathrm{Z}-\mathrm{CPS}$ & 0.103 & 0.077 & $Z_{A} \succ Z_{B}$ & Consistent \\
\hline
\end{tabular}

Table 2.Comparative results using $\mathrm{Z}$ - fuzzy numbers of Case 2 .

\begin{tabular}{lcccc}
\hline \multirow{2}{*}{ Methods } & \multicolumn{2}{c}{$Z$ - fuzzy numbers } & Ranking & \multirow{2}{*}{ Evaluation } \\
\cline { 2 - 3 } & $Z_{A}$ & $Z_{B}$ & Results & Inconsistent \\
\hline Z - C. Cheng (1998) & 0.583 & 0.583 & $Z_{A} \approx Z_{B}$ & Inconsistent \\
Z - C. Chu, C. Tsao (2002) & 0.150 & 0.150 & $Z_{A} \approx Z_{B}$ & Inconsing \\
Z - S. Chen, J. Chen (2009) & 0.258 & 0.278 & $Z_{A} \prec Z_{B}$ & Inconsistent \\
Z - A. Kumar et al. (2010) & 0.500 & 0.500 & $Z_{A} \approx Z_{B}$ & Inconsistent \\
Z - S. Chen, K. Sanguansat (2011) & 0.300 & 0.300 & $Z_{A} \approx Z_{B}$ & Inconsistent \\
Z - T. Allahviranloo, R. Saneifard (2012) & 0.240 & 0.240 & $Z_{A} \approx Z_{B}$ & Inconsistent \\
Z - L. Dat et al. (2012) & 0.111 & 0.111 & $Z_{A} \approx Z_{B}$ & Inconsistent \\
Z - V. Yu et al. (2013) for $\alpha=0$ & 1.000 & 1.000 & $Z_{A} \prec Z_{B}$ & Inconsistent \\
Z - V. Yu et al. (2013) for $\alpha=0.5$ & 1.000 & 1.000 & $Z_{A} \approx Z_{B}$ & Inconsistent \\
Z - V. Yu et al. (2013) for $\alpha=1$ & 1.000 & 1.000 & $Z_{A} \succ Z_{B}$ & Consistent \\
Z - CPS & 0.103 & 0.077 & $Z_{A} \succ Z_{B}$ & Consistent \\
\hline
\end{tabular}


Table 3.Comparative results using Z - fuzzy numbers of Case 3.

\begin{tabular}{|c|c|c|c|c|}
\hline \multirow[t]{2}{*}{ Methods } & \multicolumn{2}{|c|}{$\begin{array}{c}Z-\text { fuzzy } \\
\text { numbers }\end{array}$} & \multirow{2}{*}{$\begin{array}{c}\text { Ranking } \\
\text { Results }\end{array}$} & \multirow[t]{2}{*}{ Evaluation } \\
\hline & $Z_{A}$ & $Z_{B}$ & & \\
\hline Z - C. Cheng (1998) & $\mathrm{x}$ & $\mathrm{x}$ & - & Inconsistent \\
\hline Z - C. Chu, C. Tsao (2002) & $\mathrm{x}$ & $\mathrm{x}$ & - & Inconsistent \\
\hline Z - S. Chen, J. Chen (2009) & 0.254 & 0.258 & $Z_{A} \prec Z_{B}$ & Consistent \\
\hline Z - A. Kumar et al. (2010) & $\mathrm{x}$ & $\mathrm{x}$ & - & Inconsistent \\
\hline Z - S. Chen, K. Sanguansat (2011) & 0.300 & 1 & $Z_{A} \prec Z_{B}$ & Inconsistent \\
\hline Z - T. Allahviranloo, R. Saneifard (2012) & $\mathrm{x}$ & $\mathrm{x}$ & - & Inconsistent \\
\hline Z - L. Dat et al. (2012) & 0.333 & 1.082 & $Z_{A} \prec Z_{B}$ & Consistent \\
\hline$Z-C P S$ & 0.077 & 0.333 & $Z_{A} \prec Z_{B}$ & Consistent \\
\hline
\end{tabular}

Note: ' $x$ ' represent ranking method as unable to rank $Z$ - fuzzy numbers '-' not applicable for ranking method.

Table 4.Comparative results using $\mathrm{Z}$ - fuzzy numbers of Case 4 .

\begin{tabular}{|c|c|c|c|c|}
\hline \multirow{2}{*}{ Methods } & \multicolumn{2}{|c|}{$\mathrm{Z}$ - fuzzy numbers } & \multirow{2}{*}{$\begin{array}{l}\text { Ranking } \\
\text { Results }\end{array}$} & \multirow{2}{*}{ Evaluation } \\
\hline & $Z_{A}$ & $Z_{B}$ & & \\
\hline Z - C. Cheng (1998) & 0.583 & 0.583 & $Z_{A} \approx Z_{B}$ & Inconsistent \\
\hline Z - C. Chu, C. Tsao (2002) & -0.150 & 0.150 & $Z_{A} \prec Z_{B}$ & Consistent \\
\hline Z - S. Chen, J. Chen (2009) & -0.258 & 0.258 & $Z_{A} \prec Z_{B}$ & Consistent \\
\hline Z - A. Kumar et al. (2010) & 0 & 0 & $Z_{A} \approx Z_{B}$ & Inconsistent \\
\hline Z - S. Chen, K. Sanguansat (2011) & -0.300 & 0.300 & $Z_{A} \prec Z_{B}$ & Consistent \\
\hline Z - T. Allahviranloo, R. Saneifard (2012) & 0.150 & 0.133 & $Z_{A} \succ Z_{B}$ & Consistent \\
\hline Z - L. Dat et al. (2012) & 0 & 0.600 & $Z_{A} \prec Z_{B}$ & Consistent \\
\hline $\mathrm{Z}-\mathrm{CPS}$ & -0.077 & 0.077 & $Z_{A} \prec Z_{B}$ & Consistent \\
\hline
\end{tabular}

\section{References}

S. M. Chen, A. Munif, G-S. Chen, H - S. Liu and B - C. Kuo, Fuzzy Risk Analysis Based on Ranking Generalized Fuzzy Numbers with Different Heights and Different Spreads, Expert Systems with Applications 39 (2012) 6320-6334.

D. Wu, D. D. Wu, Y. Zhang and D. L. Olson, Supply chain outsourcing risk using integrated stochastic - fuzzy optimization approach, Information Sciences 235 (2013) 242-258.

J. D. Bermudez, J. V. Segura and E. Vercher, A multi objective genetic algorithm for cardinality constrained fuzzy portfolio selection, Fuzzy Sets and Systems 188 (1) (2012) 16-26.

S. Ebrahimnejad, S. M. Mousavi, R. T. Moghaddam, H. Hashemi, and B. Vahdani, A novel two - phase group decision making approach for construction project selection in a fuzzy environment, Applied Mathematical Modelling 36 (9) (2012) 4197-4217.

D. C. Morais and A. T. Almeida, Group decision making on water resources based on analysis of individual rankings Omega 40 (1) (2012) 42-52.
H. Deng, Comparing and ranking fuzzy numbers using ideal solutions, Applied Mathematical Modelling, (2013) in press.

H - J. Zimmermann, An application - oriented view of modelling uncertainty, European Journal of Operational Research 122 (2000) 190-198.

A. Kumar, P. Singh, P. Kaur and A. Kaur, A New Approach for Ranking Generalized Trapezoidal Fuzzy Numbers, World Academy of Science, Engineering and Technology 68 (2010) 229-302.

R. Jain, Decision-Making In The Presence of Fuzzy Variable, IEEE Transactions on Man and Cybernetic 6 (1976) 698-703.

S. M. Chen and J. H. Chen, Fuzzy Risk Analysis Based on Ranking Generalized Fuzzy Numbers with Different Heights and Different Spreads, Expert Systems with Applications 36 (2009) 6833-6842.

A. S. A. Bakar, D. Mohamad and N. H. Sulaiman, Ranking Fuzzy Numbers using Similarity Measure with Centroid, IEEE International Conference on Science and Social Research, (2010) 58-63. 
S. M. Chen and K. Sanguansat, Analyzing fuzzy risk based on a new fuzzy ranking method between generalized fuzzy numbers, Expert System with Applications 38 (2011) 2163-2171.

A. S. A. Bakar, D. Mohamad and N. H. Sulaiman, Distance - Based Ranking Fuzzy Numbers, Advances in Computational Mathematics and Its Applications 1(3) (2012) 146-150.

L. Q. Dat, V. F. Yu, and S. Y. Chou, An Improved Ranking Method for Fuzzy Numbers Based on the Centroid Index, International Journal of Fuzzy Systems 14 (3) (2012) 413-419.

V. F. Yu, H. T. X. Chi, and C. W. Shen, Ranking Fuzzy Numbers based on Epsilon - Deviation Degree, Applied Soft Computing 13 (8) (2013) 3621-3627.

A. S. A. Bakar and A Gegov, Ranking of fuzzy numbers based centroid point and spread, Journal of Intelligent and Fuzzy Systems 27 (2014) 1179-1186.

B. Kang, D. Wei, Y. Li and Y. Deng, A method of converting $\mathrm{Z}$ - numbers to classical fuzzy numbers, Journal of Information and Computational Science 9 (3) (2012a) 703-709.

L. A. Zadeh, A note on $\mathrm{Z}$ - numbers, Information Sciences 181 (2011) 2923-2932.

R. R. Yager, On A View of Zadeh's Z - Numbers, Springer-Verlag Berlin Heidelberg, (2012a) 90-101.
R. R. Yager, On Z - Valuations using Zadeh's Z Numbers, International Journal of Intelligent Systems 27 (3) (2012b) 259-278.

B. Kang, D. Wei, Y. Li, and Y. Deng,. Decision Making using Z - Numbers Under Uncertain Environemnt, Journal of Computational Information Systems 8 (7) (2012b) 2807-2814.

L. A. Gardashova, Application of Operational Approaches to Solving Decision Making Problem Using Z Numbers, Journal of Applied Mathematics 5 (9) (2014) 1323-1334.

B. S. Shieh, An Approach to Centroids of Fuzzy Numbers, International Journal of Fuzzy Systems 9 (2007) 5154.

C. H. Cheng, A New Approach for Ranking Fuzzy Numbers by Distance Method, Fuzzy Sets and System 95 (1998) 307-317.

C. T. Chu, and C. T. Tsao, Ranking Fuzzy Numbers with an Area Between the Centroid Point and Original Point, Computer and Mathematics with Applications 43 (2002) 111-117.

T. Allahviranloo and R. Saneifard, Defuzzification Method for Ranking Fuzzy Numbers based on Center of Gravity, Iranian Journal of Fuzzy Systems 9 (6) (2012) $57-67$. 